\title{
Pertinencia del contenido y desarrollo metodológico de las asignaturas tecnológicas en las titulaciones de Ciencias de la Comunicación en España
}

\author{
Luis Fernando Morales Morante \\ (Universidad Autónoma de Barcelona, España) \\ Recibido: 10/11/09 \\ Aceptado: 12/12/09
}

RESUMEN: La asignatura de Tecnología de los Medios Audiovisuales tiene un carácter fundamental en las carreras de Comunicación. Sin embargo, usualmente, no se advierte una relación directa entre la teoría, la práctica y las competencias que se pretenden desarrollar. En el presente artículo se realiza un análisis comparado de esta materia en las principales universidades españolas. Se explican las principales razones pedagógicas, estructurales y de mercado que generan estas diferencias, y finalmente se elabora un conjunto de propuestas con la finalidad de mejorar los contenidos y la dinámica docente.

Palabras clave: Docencia / formación universitaria / tecnologías / aprendizaje / Espacio Europeo de Educación Superior

\section{Technological subjects relevance in the Communication's degree in Spain: Methodological development and content relevance}

SUMMARY: The teaching of audiovisual media technology subjects is critical when refering to the Communication careers. However, a direct relation between theory, practice and powers to be developed is not usually noted. This article makes a comparative analysis of this subject, taught in the main Spanish universities. The main pedagogical market and structure reasons that generate this differences are also explained, and, at the end, a group of proposals in order to gnhace the conents and teaching dynamics are made.

Keywords: Teaching / university education / technologies / learning / superior education european space 


\section{Introducción}

T a vorágine tecnológica en la que L se ve envuelto hoy el mundo profesional y empresarial alrededor de las Ciencias de la Comunicación viene ejerciendo una fuerte influencia en el diseño e implementación de ciertas asignaturas básicas de las carreras que conforman esta rama del conocimiento, así como en los criterios logísticos de elección del equipamiento idóneo para la realización de las prácticas por parte de los centros de educación. Estos acontecimientos, finalmente, han afectado el diseño curricular general, el logro efectivo de las competencias establecidas y la formación última de un conjunto de capacidades de cara a la inserción de los alumnos en el mercado laboral. Un análisis comparativo del contenido de esta materia en las distintas facultades de comunicación en España pone de manifiesto planteamientos con un acentuado carácter técnico, operativo, de producción o incluso con una pro- fundización innecesaria de conocimientos más próximos a la ingeniería electrónica y de telecomunicaciones. De cara a la implantación definitiva de los nuevos planes de estudio, en el marco del Espacio Europeo de Educación Superior (EEES), ${ }^{1}$ resulta oportuno discutir acerca de la pertinencia y sentido real de los temarios y modelos de enseñanza de esta asignatura, tomando en cuenta su consonancia con la parte práctica y por el hecho último de que a partir de dicha formación los alumnos cursan otras materias posteriores y orientan definitivamente su proyección y capacitación futura hacia el mundo laboral.

\section{Las carreras de Comunicaciones y el uso de las tecnologías}

Históricamente, en España, la enseñanza de las titulaciones universitarias afines a la rama de las Ciencias de la Comunicación (léase: Periodismo, Comunicación Audiovisual y Publicidad y Relaciones Públicas), ${ }^{2}$ ha veni-

1 El nuevo Espacio Europeo de Educación Superior (EEES) viene implementándose progresivamente desde el 2005 y pretende desarrollar un plan de estudios común de pregrado y posgrado, entre los centros de enseñanza de la Unión Europea. Para ello se impulsa especialmente el intercambio de alumnos y profesores entre universidades. Se desarrollan políticas comunes para poner en marcha modelos didácticos y metodológicos innovadores que reducen la carga teórica presencial y otorgan mayor valor al trabajo individual y grupal del estudiante. Esto lleva a una reformulación completa del modelo de enseñanza que incluye el uso de plataformas y mecanismos de tutoría virtual permanente del concepto de crédito y los mecanismos de evaluación del temario teórico y de las sesiones de práctica presenciales.

2 Esta titulación viene funcionando en período de prueba desde el curso 2005-2006. La implantación definitiva de las tres titulaciones que conforman la rama de Ciencias de la Comunicación en el Modelo Europeo se implementará en el curso 2010-2011. 
do adaptando e incorporando con un más que significativo desfase temporal el uso de equipamientos y tecnologías de producción de mensajes, como complemento fundamental de la formación práctica de los estudiantes. Las demandas de un mercado laboral en constante cambio, junto con las expectativas de los estudiantes por formarse en el dominio de ciertas habilidades operacionales, por sobre la formación teórica tradicional y fundamental, ha condicionado en buena medida las decisiones recientes de los centros superiores de enseñanza españoles respecto de sus diseños de plan de estudios y la elección de las herramientas idóneas para la producción y la capacitación práctica de los alumnos. Se trata, por consiguiente, de un esquema donde realmente el tipo de equipamiento termina influyendo en la distribución entre horas de teoría, práctica y sus contenidos, así como en la metodología docente del conjunto de asignaturas fundamentales de las comunicaciones, como por ejemplo: lenguajes, géneros, producción y realización, entre otras, donde resulta indispensable la utilización de dispositivos especializados. Y esta relación se hace más evidente porque, además, en muchos casos, la dinámica docente termina siendo un factor dependiente o incluso subordinado a dichas características, al volumen de equipamiento, los espacios disponibles y su adecuación al planteamiento específico de las prácticas. El debate es intenso desde hace años. Sobre el particular, el eje de las discusiones ha girado alrededor de la conveniencia de adquirir o no equipo profesional para formar a los estudiantes. Según los académicos, es más pertinente el uso de equipo semiprofesional; para ello, argumentan una mayor sencillez para su manejo (y por ende, facilidad para explicar su funcionamiento correcto), su fortaleza para un uso intenso de horas por día y por muchos alumnos inexpertos, su acoplamiento exacto para ejercicios prácticos elementales y por su bajo coste, comparado con las tecnologías de punta. Asimismo, esta visión responde a un planeamiento logístico que impulsa la incertidumbre por contar con equipos de calidad broadcast de última generación. La rapidez con la que aparecen, se instauran y son sustituidos por otros en el mercado, hace prácticamente imposible que la universidad pueda estar "al día" con la tecnología, porque ciertamente posee un sistema poco ágil y burocrático para la renovación de sus infraestructuras. Si bien es cierto, el equipamiento empleado en la formación puede ser diferente al que en definitiva utilizará el estudiante cuando ingrese en el mercado ocupacional, el conocimiento adquirido en las aulas le será suficiente para controlar sus principios operativos y adaptarse posteriormente en el uso de las nuevas herramientas que irán apareciendo. Por el contrario, quienes proceden del mundo profesional, in- 
tentan importar directamente al terreno formativo universitario la tecnología dominante utilizada en los medios de referencia o simplemente donde ellos mismos trabajan: diarios, estaciones de radio, productoras de vídeo, cine y canales de televisión. Bajo esta filosofía, el alumno será competente tanto en el manejo instrumental como en las rutinas que la propia inmersión tecnológica y el trabajo profesional imponen. Más adelante, cuando culmine la carrera, este dominio le será indispensable para desempeñarse eficientemente en cualquier medio de comunicación. Este segundo modelo, además, convierte la dinámica docente y la practica en una tarea más sencilla, porque simplemente consiste en reproducir el sistema cotidiano de trabajo del mundo profesional.

En un contexto pedagógico más amplio y actual, son muchas las propuestas por incorporar o repotenciar, de manera diversa, el uso de recursos tecnológicos en la enseñanza superior universitaria (Rodríguez 2009, Fandos 2009, Cabrera y González 2006, Cabero et al. 2003: 81-100). Diversos estudiosos defienden esta tesis basándose principalmente en la existencia actual de una generación de nativos tecnológicos en las aulas, factor que, de hecho, obliga a replantear y actualizar contenidos, la dinámica docente, los modelos de interacción de la formación, así como el desarrollo de nuevas capacidades y actitudes renovadas del profesorado, para explicar con autoridad las innovaciones y el funcionamiento de dispositivos y procesos complejos (Aguaded 1996).

El sentido de esta nueva filosofía ha sido certeramente incorporado en el planteamiento de los nuevos planes EEES. El modelo, más conocido como "Boloña", 3 además de refundar por completo los protocolos tradicionales de la relación profesor-alumno, mediante un sistema abierto enseñanzaaprendizaje, de tutoría permanente y una fuerte carga de trabajo individual y grupal fuera de las aulas; pretende, además, adecuar la metodología práctica siguiendo un planteamiento más dinámico, afín con la lógica laboral y consecuente con las expectativas del propio estudiante y los centros respecto del mercado. Es justamente a raíz de la incorporación de estos nuevos componentes del circuito pedagógico que una parte significativa del sector académico tradicional ha fundamentado sus críticas al modelo. Se aduce que la reducción de horas de teoría acarrea una inevitable falta de profundización en ciertos aspectos conceptuales fundamentales, que el alumno, por sí solo, no sería capaz de controlar de manera suficiente en un

3 Los lineamientos principales del nuevo Espacio de Educación Superior Europeo pueden verse en la página web del Ministerio de Educación de España: <http://www.queesbolonia.es/>. 
diseño donde la carga teórica se reduce a sesiones magistrales, dejando el peso más fuerte del trabajo y la evaluación a la realización de las lecturas y actividades fuera del horario lectivo.

Pero, en tanto implantación progresiva, los planes de estudio de todas las ramas (donde se incluyen las tres carreras que forman la plataforma de las Comunicaciones) aún se encuentran en fase de elaboración o bien de ajuste para su implementación definitiva en gran parte de las universidades de España y Europa. Este escenario inusual de transición de cambio curricular es ciertamente una oportunidad para abrir un espacio de debate académico y analizar lo que se está haciendo actualmente y las pretensiones de cara al futuro. En este aspecto, el presente estudio propone una serie de pautas para mejorar aspectos formales o estructurales presentes en los programas actuales. En su sentido último, el resultado que pretendemos con este trabajo es contribuir a la señalización de un conjunto de pautas y elementos de referencia mediante los cuales se puede diseñar, implementar y monitorear una asignatura (o un tipo de asignatura fundamentada en una base tecnológica), partiendo del principio de coherencia interna, por su contenido, en consonancia con otras asignaturas y las competencias fundamentales que señala el perfil general de la titulación.

\section{Mallas y contenidos: Qué se enseña y cuándo se enseña}

Pero qué está sucediendo realmente con estas asignaturas. ¿Se enseña realmente tecnología de la comunicación audiovisual como se señala en la mayoría de las titulaciones? ¿Se observan cambios importantes en las nuevas propuestas de plan de estudios? ¿Existe actualmente coherencia entre el temario de teoría y la parte práctica o más bien esta última continúa decantándose por la enseñanza del manejo operativo de los equipos de registro y del tratamiento de imagen, sonido y montaje? Esta confusión entre el nombre, los contenidos y el diseño metodológico es muy frecuente $y$, a nuestro parecer, representa el núcleo desde el cual se impulsa una formación muchas veces inconexa e inadecuada de los estudiantes, porque no suministra el conocimiento apropiado y tampoco se desarrolla manteniendo un sentido lógico, es decir, como estudio previo de otras materias igualmente fundamentales de estas tres carreras: lenguaje, géneros y realización, como debería de ocurrir.

Seguidamente, efectuaremos un análisis comparativo del diseño de esta asignatura en las diferentes titulaciones de las carreras de Comunicaciones.

En la siguiente tabla podemos observar la configuración de la asignatura en la titulación de Comunicación Audiovisual, atendiendo a su localización en el plan curricular, su du- 
ración en horas, ${ }^{4}$ ciclos, créditos y su carácter troncal u obligatorio. Hemos focalizado el análisis en las universidades públicas más representativas de España, según su antigüedad, reconocimiento académico, volumen de alumnos y el posicionamiento efectivo de sus egresados en el mercado laboral.

En la titulación de Comunicación Audiovisual, la asignatura se ubica en el primer o segundo curso. En todas las universidades estudiadas es prerrequisito para cursar Lenguaje o Narrativa Radiofónica y Televisiva, Géneros, Producción y Realización de Programas. Únicamente en la Universidad de Málaga, el nombre queda sustituido por Comunicación e Información Audiovisual, pero el conteni- do se centra igualmente en el funcionamiento de las tecnologías de producción de mensajes en diferentes soportes. En cuanto al carácter, solo en las universidades de Sevilla y Málaga es troncal y en todas las restantes es de carácter obligatorio. La duración representa un factor más variable. Dependiendo de la facultad, la programación puede ser cuatrimestral, semestral o anual. Este hecho lleva a que si efectivamente el contenido temático es similar, entonces la variación en la carga horaria semanal y del curso en su totalidad, puede terminar influyendo fuertemente en el nivel de ampliación o profundización de los distintos puntos del temario y la programación. Los alumnos que tienen en este caso una carga horaria mayor son

Tabla 1

Comunicación Audiovisual

\begin{tabular}{lclccl}
\hline Universidad & Curso & Duración & $\begin{array}{c}\text { Créditos } \\
\text { Horas T/P }\end{array}$ & $\begin{array}{c}\text { Total de } \\
\text { horas }\end{array}$ & Carácter \\
\hline Autónoma de Barcelona (UAB) & 1 & Semestral & $5 / 1-2$ & 48 & Obligatoria \\
Complutense de Madrid (UCM) & 2 & Anual & $12 / 4$ & 128 & Obligatoria \\
Carlos III (CA) & 2 & Cuatrimestral & $6 / 1,5+4,5$ & 96 & Obligatoria \\
Sevilla (US) & 1 & Anual & $12 / 4$ & 136 & Troncal \\
Málaga (UM) & 1 & Cuatrimestral & $8 / 4+4$ & 80 & Troncal \\
Politécnica de Valencia (UPV) & 1 & Semestral & $6 /$ ETS & 131 & Obligatoria \\
Santiago de Compostela (USC) & 1 & Semestral & $6 /$ ETS & 51 & Obligatoria \\
\hline
\end{tabular}

Fuente: Planes de estudio oficiales de las universidades que ofrecen la asignatura en esta titulación. Elaboración propia.

4 Las palabras semestral o cuatrimestral diferencian la nomenclatura académica en las comunidades autónomas para denominar, en ambos casos, un período de 16 semanas de clases lectivas. 
los de la Universidad de Sevilla con 12 horas por semana, le sigue la de Málaga con 8 y luego la Complutense de Madrid con 4. Finalmente, los que dedican más horas a lo largo de la asignatura (horas semanales multiplicadas por el número de semanas del curso) son la Universidad de Sevilla con 136 horas, seguida por la Complutense con 128 y muy de cerca por la Politécnica de Valencia con 131. Más alejada está la de Málaga con 80, mientras que las de menor carga son Carlos III con 96 horas, Santiago de Compostela con 51 y Autónoma de Barcelona con 48 .

A continuación procedemos a analizar, por separado, el contenido y la metodología en cada una de las universidades, atendiendo a la orientación específica de las sesiones prácticas y su relación con la teoría.

En la UAB, la teoría se centra en los principios básicos de audio y video, los equipos de producción y los modos de transmisión de señales. Para las prácticas se crean tres grandes módulos, por donde los alumnos circulan en 6 o 7 grupos de entre 5 y 6 personas cada uno. Las sesiones tienen como objetivo central una primera aproximación al manejo del estudio y las herramientas de grabación y edición de audio digital (Módulo Radio), el manejo de la cámara ENG y el plató de televisión (Módulo TV) y, finalmente, del sistema de edición no lineal de vídeo (Módulo AVID).
La asignatura en la UCM desarrolla, en la teoría, el protocolo de señales y el fundamento de la tecnología digital; también los formatos de imágenes digitales, vídeo, multimedia y los códecs. Se realizan prácticas de plató, laboratorio digital de imagen y sonido, en grupos de 8 personas. Para las prácticas se divide el grupo en 3 turnos de 40 alumnos cada uno. Estos, a su vez, son subdivididos en grupos de 2 alumnos por cada puesto informático. La dedicación de la asignatura es de 4 horas por semana. Efectúan prácticas rotativas sin opción a cambio en las aplicaciones de diseño fotográfico PhotoShop y de edición no lineal en la plataforma Adobe Premier.

La Universidad Carlos III enfoca la asignatura en torno a la explicación de técnicas y formatos de imagen y sonido. Las prácticas incluyen ejercicios de producción y realización de pequeñas piezas, con un importante número de horas de edición de vídeo.

En la US el temario se orienta a la producción y el lenguaje. Se explica el manejo de la cámara y la señal de televisión. La grabación y la edición de audio, vídeo y cine. Para los ejercicios se efectúa una subdivisión por grupos donde se explica en teoría y grupos pequeños su funcionamiento. Se planifican un total de 13 prácticas de campo, que conllevan tanto el adiestramiento en el funcionamiento como la producción de algunas pequeñas piezas en vídeo. 
La UM centra el temario teórico preferentemente en la imagen. Se abordan temas de física: naturaleza de la luz, el color y la óptica, así como las diferencias entre lo analógico y la señal digital, así como los modos de transmisión de televisión. Incluye también trabajos prácticos de realización, en este caso entrevistas, y la preparación de una breve secuencia de ficción. Como información adicional, la planificación del curso también contempla la visita de los alumnos a un canal de televisión local y una sesión sobre videoarte.

Por su parte, el programa adaptado al nuevo modelo de educación superior de la Universidad Politécnica de Valencia añade, principalmente, una prospección de las nuevas tecnologías: sistemas de comunicación, TV color y alta definición, TV digital y realidad virtual. La metodología incluye exposición de contenidos y presentación de un proyecto desarrollando un problema. Las prácticas de laboratorio se restringen a la cámara y funcionamiento del plató. No se incluyen prácticas de edición. Finalmente, en la USC se explica el manejo y las diferencias entre las cámaras de cine y de televisión. Las tecnologías del sonido, de iluminación y las bases de logísticas de la posproducción. Se pretende que los estudiantes sean capaces de operar solos cada uno de los sistemas, siguiendo lo aprendido en las clases teóricas. Se programan 14 prácticas en las cuales se incluye el manejo de la cámara de vídeo y algunas pautas básicas de sonido y edición.

Los contenidos teóricos más completos se encuentran probablemente en la UM y la UPV, aun cuando, nuevamente, son importados de Comunicación Audiovisual. Se explican los fundamentos de percepción, la colorimetría, un módulo de cine electrónico, un esbozo de la revolución digital y las pautas básicas de revelado de películas. La UPV es la única facultad donde el programa incluye sesiones de análisis y elaboración de procesos tecnológicos audiovisuales. Otro tema novedoso lo ofrece la UM al incluir el vídeoarte junto con técnicas y procesos de la materialización de la información con presencia de profesionales y expertos invitados. Finalmente, en la USC la orientación se enfoca en el aspecto operativo. Solo las semejanzas y diferencias entre cine y televisión se supeditan a la explicación de los procedimientos y mecanismos de los diferentes sistemas de grabación analógica y digital. En el resto de temas hay un predominio de las tecnologías del sonido y la posproducción de programas, con fuerza en el uso semántico de los efectos de montaje, la inserción de títulos y, en general, de los aportes de la informática en las tareas de posproducción.

Veamos ahora cómo se definen los mecanismos de evaluación. En la UAB los docentes evalúan y penalizan los indicadores de imprecisión operativa; por ejemplo, los errores en el 
manejo de la cámara, la captura y la conversión de formatos, los cortes y las transiciones de edición. La asistencia regular a las clases de teoría compensa la evaluación global. En términos porcentuales de la nota, la realización de las prácticas de campo tiene un peso de $75 \%$ y el examen teórico el $25 \%$ restante. En la UPV y la USC la evaluación se obtiene del examen teó- rico: $40 \%$, los trabajos de las prácticas: $40 \%$, y el $20 \%$ restante de la actitud. En las otras universidades: UCM, US y UM, la nota final se obtiene de la media entre teoría y práctica. Para superar la materia es necesario un mínimo de 5 sobre 10 y ambas partes deben ser aprobatorias en todos los casos.

Tabla 2

Titulación de Comunicación Audiovisual: Objetivos y planificación teórico-práctica por universidades

\begin{tabular}{lll}
\hline Universidad & Objetivos/temario teórico & Sesiones prácticas \\
\hline $\begin{array}{l}\text { Autónoma de } \\
\text { Barcelona }\end{array}$ & $\begin{array}{l}\text { Principios y fundamentos básicos de } \\
\text { audio y video. Señal analógica y digital. } \\
\text { Equipos de producción de vídeo: la } \\
\text { cámara. Equipos de producción de } \\
\text { audio: la mesa de mezcla. La edición de } \\
\text { vídeo. Modos de transmisión de señales: } \\
\text { terrestre, cable y satélite. }\end{array}$ & $\begin{array}{l}\text { Funcionamiento del plató de TV, cáma- } \\
\text { ras ditales, edición Avid, grabadoras }\end{array}$ \\
\hline $\begin{array}{l}\text { Complutense de radio. } \\
\text { de Madrid }\end{array}$ & $\begin{array}{l}\text { La señal de vídeo, tecnología digital: for- } \\
\text { matos y comprensión de cine, redacción } \\
\text { digital: sistemas y servicios. Formatos de } \\
\text { imágenes digitales, vídeo y multimedia, } \\
\text { códecs. }\end{array}$ & \\
\hline en Adobe Premier. \\
\hline
\end{tabular}

(continúa) 
(continuación)

\begin{tabular}{lll}
\hline Universidad & Objetivos/temario teórico & Sesiones prácticas \\
\hline \multirow{2}{*}{ Sevilla } & La cámara y la señal de vídeo. Introduc- & Realización de un corto, y de un progra- \\
& ción al lenguaje, edición de vídeo. El & ma radial, planos e iluminación. \\
& montaje, la luz y el color. Iluminación y & \\
& grabación de vídeo. Formatos. El estudio \\
& de TV. Equipos ENG. Instrumentos y téc- \\
& nicas radiofónicas. Introducción al len- \\
& guaje. El sonido. Grabación y edición de \\
& audio. El proceso cinematográfico, el so- \\
& nido y el montaje.
\end{tabular}

Málaga Equipos y sistemas de trabajo. Naturaleza de la luz y el color. Óptica. Imágenes analógicas. Registro. lluminación. Edición electrónica. Señales digitales. Transmisión de televisión. Sonido.
Realización de una entrevista en directo, informativo, promoción en directo y una secuencia de ficción.
Politécnica de Valencia
Historia y evolución de la radio y la televisión. Sistemas de comunicación, sonido, vídeo y teledifusión. TV color y alta definición. TV digital, realidad virtual. Sistemas de TV por cable y satélite. Sistemas de almacenamiento. Plató de televisión.
Prácticas de grabación, tanto de imagen como sonido en mesa de mezclas.
Santiago de Compostela
La cámara de cine y la cámara de TV. Manejo del trípode, lente de cámara de Tecnología del sonido. Iluminación cine- TV, captación de sonido con micros. Ilumatográfica, televisiva y de espectáculos. La emisora de televisión. Tecnología informática aplicada a la posproducción. minación. Estudio y control TV. Edición básica, titulación y efectos.

Fuente: Información de los programas oficiales de la asignatura en cada universidad. Elaboración propia.

Analizando los contenidos y ejercicios prácticos, por ejemplo, en la US, la Carlos III y la UM existe un diseño dual, que pretende poner en marcha tanto el conocimiento de la operación de equipos como de lenguaje y narrativa audiovisual, aun cuando estos úl- timos no logren desarrollarse suficientemente en el temario teórico y serán profundizados en asignaturas posteriores de la carrera. Esto, a nuestro parecer, es negativo, porque finalmente el estudiante estará al frente de una duplicidad de temas y de una 
nueva e innecesaria disonancia teórico-práctica, que puede producir un malestar y desánimo respecto de sus expectativas de formación. No obstante, considerando el número de horas efectivas de la asignatura en estas universidades, es probable que la decisión obedezca sencillamente al número total de horas de clase y a una mayor cuota práctica de inmersión. En otro aspecto particular, en la UCM, la UPV y la Carlos III hay sesiones de teoría dedicadas a la tecnología de producción del cine, aunque las prácticas se hacen siempre en soporte videográfico, el resto de facultades prescinde directamente de estos contenidos porque carecen del equipo idóneo para las prácticas.

Como se distingue en la segunda y tercera columnas de la tabla, hay un permanente divorcio teórico-práctico, debido a que una parte importante del contenido no se complementa directamente con una actividad práctica, y el sistema modular Radio, TV, Edición, por grupos (UAB, UPV y Carlos III), si bien es útil por ofrecer un primer contacto de las tecnologías agrupadas por espacios de trabajo y dinámicas organizativas, lleva a una inevitable alteración del suministro de los contenidos, habida cuenta de que los alumnos, al estar ordenados por grupos-módulos, van desarrollando sus prácticas en función del acceso a los espacios y no por la relación con la carga teórica. Sin embargo, la fragmentación permite un mejor con- trol de los grupos y la creación de una dinámica sumamente controlada de las explicaciones, aun cuando no siempre existan prácticas puntuales de iluminación o de formatos de emisión. Esta limitación ha tratado de controlarse en el plan de la UCM, al estipular las prácticas únicamente en el proceso de registro de información de vídeo digital, con énfasis en los formatos y códecs. Da preferencia a una mayor inmersión en dos tecnologías básicas, pero impide un contacto con otras plataformas. En la US se pretende articular de forma coherente una explicación previa, con menos grupos y un mayor número de semanas que se enlace mejor con los ejercicios. Se realizan además sesiones concretas de producción de material audiovisual, aun cuando estas prácticas se repetirán nuevamente en asignaturas posteriores.

Como puede comprobarse, los contenidos y la estructuración de las prácticas es muy variada y no siempre responden al perfil general que se pretende definir en cada grado o título, de acuerdo con los lineamientos académicos que estipula la legislación marco del Ministerio de Educación, sino que, internamente, acaban ejecutando estructuras de prácticas e impulsando competencias diferentes, según sus verdaderas capacidades logísticas y de profesorado. No obstante, el modelo de evaluación de la nota media entre teoría y práctica es claramente el más utilizado por los docentes, salvo en las 
titulaciones no implementadas en el nuevo modelo Boloña. En este caso, ambas notas deben ser necesariamente aprobatorias.

Una vez desgranados los programas de Comunicación Audiovisual, veamos cómo se articulan estos mismos elementos en la titulación de periodismo:
Como se muestra en la segunda columna de la tabla, la asignatura se ubica en el primer o segundo curso de la titulación. En todas las universidades es prerrequisito para cursar Lenguaje Radiofónico, Televisivo, Géneros, Rutinas, y Producción y Programas Informativos. En cuanto al carácter, en la UAB y la USC es obligatoria,

Tabla 3

Periodismo

\begin{tabular}{lclcrl}
\hline Universidad & Curso & Duración & $\begin{array}{c}\text { Créditos } \\
\text { Horas }\end{array}$ & $\begin{array}{c}\text { Total de } \\
\text { horas }\end{array}$ & Características \\
\hline Autónoma de Barcelona & 1 & Semestral & $5 / 1-2$ & 48 & Obligatoria \\
Complutense de Madrid & 2 & Anual & $12 / 4$ & 128 & Troncal \\
Sevilla & 1 & Anual & $12 / 4$ & 128 & Troncal \\
Málaga & 2 & Cuatrimestral & $8 / 4+4$ & 80 & Troncal \\
País Vasco & 1 & Cuatrimestral & $6 /$ ETS & 45 & Troncal \\
Santiago de Compostela & 1 & Semestral & 6/ETS & 51 & Obligatoria \\
\hline
\end{tabular}

Fuente: Información obtenida de los planes de estudios de las universidades que ofrecen la titulación. Elaboración propia.

en las demás es troncal. En la UCM y la US es anual, mientras que en las restantes es semestral/cuatrimestral. Este indicador, al ser relacionado, da ratios de dedicación horaria variables. Así, los alumnos que tienen una carga horaria más elevada son los de la US y la UCM con un total de 128 horas. Le sigue la UM con 80 . Las que tienen menos dedicación son la USC, la UAB y la UPV con 51, 48 y 45 horas, respectivamente. Este modelo estructural crea finalmente desbalances muy pronunciados en la formación y el número definitivo de horas dedicadas a cada módulo y dispositivo. Por ejemplo, un alumno de la UCM o la US destina más del doble de horas comparado con uno formado en la USC, la UAB o la UPV.

Seguidamente, veamos más de cerca los temarios para determinar cómo estas diferencias afectan el desarrollo y la coherencia entre la teoría y la práctica en cada una de las titulaciones o grados. 
Tabla 4

Titulación de periodismo: Objetivos y planificación teórico-practica por universidades

\begin{tabular}{|c|c|c|}
\hline Universidad & Descriptor/Temario & Contenido práctico \\
\hline Autónoma de Barcelona & $\begin{array}{l}\text { Principios básicos de audio y ví- } \\
\text { deo. Fundamentos tecnológicos. } \\
\text { Señal analógica y digital. Equipos } \\
\text { de producción de vídeo: la cámara. } \\
\text { Equipos de producción de audio: la } \\
\text { mesa de mezcla. La edición de ví- } \\
\text { deo. Modos de transmisión de se- } \\
\text { ñales: terrestre, cable y satélite. }\end{array}$ & $\begin{array}{l}\text { Funcionamiento del plató de } \\
\text { TV, cámaras, edición Avid y } \\
\text { estudio de radio. }\end{array}$ \\
\hline Complutense de Madrid & $\begin{array}{l}\text { Concepto de la tecnología de la } \\
\text { información. } \\
\text { Evolución tecnológica en la crea- } \\
\text { ción de información escrita. Tecno- } \\
\text { logía del diseño periodístico de in- } \\
\text { formación escrita y digital. }\end{array}$ & $\begin{array}{l}\text { Herramientas de tecnologías } \\
\text { de información y redacción } \\
\text { escrita. }\end{array}$ \\
\hline Sevilla & $\begin{array}{l}\text { La cámara y la señal de vídeo. In- } \\
\text { troducción al lenguaje. Edición de } \\
\text { vídeo y montaje. La luz y el color. } \\
\text { lluminación. Grabación y formatos. } \\
\text { Estudio de TV, equipos ENG. Ins- } \\
\text { trumentos y técnicas radiofónicas. } \\
\text { Introducción al lenguaje del sonido, } \\
\text { grabación y edición de audio. Pro- } \\
\text { ceso cinematográfico, el sonido, el } \\
\text { montaje. }\end{array}$ & $\begin{array}{l}\text { Realización de un corto, un } \\
\text { programa radial en Pro Tools, } \\
\text { planos e iluminación. }\end{array}$ \\
\hline Málaga & $\begin{array}{l}\text { Fenómenos ondulatorios. La cap- } \\
\text { tación analógica. Montaje, modos. } \\
\text { Cine digital. Imágenes sonoras y } \\
\text { electrónicas. TV, cámaras, en co- } \\
\text { lor, luminancia y crominancia. El } \\
\text { estudio de televisión. El vídeo-arte. }\end{array}$ & $\begin{array}{l}\text { Técnicas y procesos de la } \\
\text { materialización de la informa- } \\
\text { ción en soportes y medios de } \\
\text { comunicación. Invitación de } \\
\text { profesionales expertos. }\end{array}$ \\
\hline
\end{tabular}




\begin{tabular}{|c|c|c|}
\hline Universidad & Descriptor/Temario & Contenido práctico \\
\hline País Vasco & $\begin{array}{l}\text { Formación y registro de la imagen, } \\
\text { propiedades de la luz, ojo humano. } \\
\text { Sonido. Color. Película. Grabación. } \\
\text { Laboratorio de revelado. Cine elec- } \\
\text { trónico y revolución digital. Televi- } \\
\text { sión. Difusión Magnetoscopios. }\end{array}$ & $\begin{array}{l}\text { Análisis y elaboración de pro- } \\
\text { cesos tecnológicos audiovi- } \\
\text { suales. }\end{array}$ \\
\hline Santiago de Compostela & $\begin{array}{l}\text { La cámara de cine y la cámara de } \\
\text { TV. Tecnología del sonido. llumina- } \\
\text { ción cinematográfica, televisiva y } \\
\text { de espectáculos. La emisora de } \\
\text { TV. } \\
\text { Tecnología informática aplicada a } \\
\text { la posproducción. }\end{array}$ & $\begin{array}{l}\text { Manejo del trípode, lente de } \\
\text { la cámara de TV, captación } \\
\text { de sonido. Mesa de ilumina- } \\
\text { ción. Estudio y control de TV. } \\
\text { Edición básica. Titulación y } \\
\text { efectos. }\end{array}$ \\
\hline
\end{tabular}

Fuente: Información obtenida de los planes de estudios de las universidades que ofrecen la titulación. Elaboración propia.

En la UAB, el contenido teórico es el mismo que en la CA y aborda temas sobre el funcionamiento de dispositivos, audio y vídeo, formas de transmisión y las diferencias fundamentales entre el sistema analógico y digital. El esquema de prácticas es de 3 módulos (Radio, TV y Edición) con 4 sesiones cada uno y grupos de 6 o 7 estudiantes.

La teoría desarrolla contenidos estrictamente tecnológicos de forma general; no obstante, atendiendo esta discordancia, existen sesiones en las que se explica el funcionamiento específico de los diferentes aparatos para las prácticas con el apoyo de guías, manuales y protocolos de manejo preparados especialmente para los alumnos.

En la UCM, el temario teórico y las prácticas están mejor relacionados y se trabaja en software especializado en la diagramación de medios impresos y digitales. El sistema orienta la formación teórica y práctica directamente hacia el uso de herramientas creadas para la generación de contenidos en prensa tradicional y digital. Intencionalmente se descartan contenidos relacionados con la grabación en plató, los cuales pasan a ser repertorio exclusivo de la titulación de Comunicación Audiovisual. Como ya es propio, la dinámica docente contempla sesiones teóricas con todo el alumna- 
do y una subdivisión en grupos de trabajo (en este caso 7 alumnos de secundaria) para las estaciones informáticas especializadas.

Los programas US, UM y USC son reproducciones o adaptaciones de Comunicación Audiovisual; por tanto, tienen una dosis importante de ejercicios de realización con énfasis en la ficción. Se construye un mismo modelo de distribución por espacios-módulos donde los alumnos entran en contacto con el manejo del equipamiento, pero no de sus fundamentos tecnológicos sino únicamente operacionales. Este diseño genera una fuerte incompatibilidad teórico-práctica y, por consiguiente, se orienta hacia una enseñanza de principios netamente audiovisuales, sin articular una ligazón con la dinámica periodística en la cual trabajarán los alumnos en el futuro. Aun cuando este tipo de diseño sea pedagógicamente poco relacionado con sus perfiles, las prácticas de ficción en vídeo y radio pueden ser altamente motivadores para los estudiantes, por la experiencia que supone realizar por única vez una historia de creación propia no periodística y aproximarse directamente a las diferentes funciones del quehacer audiovisual.

Respecto de los criterios de evaluación, en la UAB, cada uno de los módulos incluye ejercicios puntuables y la nota de prácticas se obtiene de la media de estos con el examen de teoría. En el caso de Periodismo el nivel de exigencia para las prácticas es lige- ramente inferior a Comunicación Audiovisual. La valoración se realiza principalmente de la ejecución correcta de los diferentes ejercicios, pero sin exigir un dominio perfecto de la herramienta. Se trata de un primer acercamiento a las infraestructuras propias del quehacer periodístico audiovisual, pero sin la profundidad propia del licenciado en Comunicación Audiovisual.

En la UCM el sistema de evaluación se obtiene de la nota media de los dos ejercicios prácticos (uno por cada módulo) y el examen final teórico, consistente en una prueba objetiva tipo test. En la US, la UM y la UPV la evaluación de la asignatura se obtiene de la nota media entre la nota de teoría y la de los diferentes ejercicios prácticos realizados durante el curso.

Con excepción del programa de la UCM, en todas las universidades el planteamiento teórico general es el mismo de Comunicación Audiovisual. Sin embargo, en la UCM, al existir un diseño con dos grandes módulos, se logra efectivamente una mayor profundización de ambos dispositivos; no obstante, cae en su propia limitación e impide la familiarización con otras herramientas igualmente valiosas para el desempeño profesional y la producción de piezas informativas especializadas. Las diferencias más notorias se encuentran en las sesiones prácticas y dejan apreciar determinadas preferencias. Resulta un tanto inesperado el hallazgo tan desi- 
gual de contenidos relacionados con las tecnologías del cine y las técnicas de montaje o edición (UM, US), cine digital y vídeoarte (UM), revelado digital (UPV), conocimientos periféricos poco útiles para el dominio propio del periodista. No hay una dedicación suficiente en explicar las diferencias entre lo analógico y lo digital. Tampoco hay programa donde se expliquen las tecnologías utilizadas en los diferentes procesos de comunicación interactiva hombre-máquina (contenido impostergable en los nuevos planes del 2010).

Posiblemente estas lagunas sean simplemente un reflejo de la falta de actualización del temario, infraestructuras especializadas o simplemente la intencionalidad por dar una formación tecnológica común en ambas titulaciones.

Finalmente, conozcamos lo que sucede en la titulación de Publicidad y Relaciones Públicas.

\section{Publicidad y Relaciones Públicas}

Como se ha mencionado, en España y el resto de Europa esta carrera viene funcionando desde el 2005 dentro de las titulaciones del Plan Piloto del modelo del Espacio Europeo de Educación Superior (EEES); por tanto, es evidente la presencia de diferencias correctivas y actualizaciones respecto de los planes denominados "antiguos", considerando que tiene un tiempo de puesta en marcha menor en comparación con las otras dos titulaciones analizadas previamente.

Veamos el planteamiento general. En la US y la UPV se dicta en el primer curso y se plantea con un criterio evolutivo; por ende, es prerrequisito de todas las demás asignaturas de la línea que marca la especialidad de comunicación audiovisual. En la UM se ubica en el segundo curso. Le sigue a Teoría y Técnica de la Imagen Fija y es prerrequisito de Producción y Realización. En la UAB se localiza en el último curso y se realiza posterior-

Tabla 5

Titulación de Publicidad y Relaciones Públicas

\begin{tabular}{lclrrl}
\hline Universidad & Curso & Duración & $\begin{array}{c}\text { Créditos } \\
\text { horas }\end{array}$ & $\begin{array}{r}\text { Total de } \\
\text { horas }\end{array}$ & Características \\
\hline Autónoma de Barcelona & 4 & Semestral & $5 / 3$ & 48 & Obligatoria \\
Sevilla & 1 & Anual & $12 / 4$ & 128 & Troncal \\
País Vasco & 1 & Cuatrimestral & $6 /$ ETS & 45 & Troncal \\
Málaga & 2 & Cuatrimestral & $8 / 4+4$ & 128 & Troncal \\
\hline
\end{tabular}

Fuente: Información obtenida de los planes de estudios oficiales de las universidades que ofrecen la titulación.

Elaboración propia. 
mente a los cursos de Redacción y Locución, y de Teoría y Técnica del Lenguaje Radiofónico y Televisivo. En cuanto al carácter, solo en la UAB es obligatoria y en las demás es troncal. En la US es anual y en las restantes es semestral/cuatrimestral. Observando la carga general, los alumnos de la US y la UM tienen finalmente más horas de clase, con un total de 128 al año. Le sigue la UAB con 48 y la UPV con 45 por semestre. Así, un alumno de la UCM o la US tienen finalmente más del doble de horas de clases comparado con uno de la UAB o la UPV.

Seguidamente, veamos más de cerca los temarios para determinar cómo el desarrollo, la conexión entre la teoría y la práctica, y el orden secuencial de los temas del programa.

Tabla 6

Titulación de Publicidad y Relaciones Públicas: Objetivos y planificación teórico-práctica por universidades

\begin{tabular}{lll}
\hline Universidad & Contenido teórico & Planeamiento de prácticas \\
\hline Autónoma de Barcelona & El proceso de producción y organi- & Funcionamiento del plató de TV, \\
& zación. Plan de trabajo y rodaje. cámaras, edición Avid y estudio de \\
& El guión publicitario: el story board radio. \\
& y el animatic. La realización y la \\
& composición visual. Posproduc- \\
& ción audiovisual digital. Los forma- \\
& tos televisivos.
\end{tabular}

Sevilla

Málaga
La cámara de vídeo. Introducción al lenguaje audiovisual. Edición de vídeo. El montaje. La luz y el color. lluminación. Grabación de vídeo. Formatos. El estudio de TV y las unidades móviles. Transmisión de señales. Instrumentos y técnicas radiofónicas. Introducción al lenguaje radiofónico. El sonido. Grabación y edición de audio. El proceso cinematográfico.
Manejo de equipos de grabación y edición. Realización de un corto, un programa radial, un spot, planos e iluminación
Capacitación práctica en el uso de las técnicas y operaciones básicas de la tecnología de la comunicación audiovisual.

Fuente: Información obtenida de los planes de estudios oficiales de las universidades que ofrecen la titulación.

Elaboración propia. 
En la UAB la asignatura desarrolla íntegramente la producción de una pieza publicitaria audiovisual en todas sus etapas y niveles: gestión de recursos humanos, artísticos, económicos y tecnológicos, tutorizada por el docente. Además, los alumnos deben presentar periódicamente un conjunto de ejercicios de análisis de anuncios mediante fichas codificadas, guiones, story boards y animaciones. La cuota tecnológica se reduce a clases prácticas vigiladas del manejo del sistema de edición no lineal, donde se hará el montaje del vídeo del trabajo final de la asignatura.

En la US y la UM se repite exactamente el diseño de Comunicación Audiovisual y Periodismo. Se enseña el manejo básico de las herramientas de grabación y tratamiento de archivos en radio y TV. La parte práctica incluye la grabación y la edición de un cortometraje en vídeo, sin especificar si se trata de una pieza publicitaria o de otro género.

En lo concerniente a la evaluación, en la UAB el ejercicio en realización del vídeo tiene valor del $50 \%$, mientras que el resto de la nota se obtiene de la media de las lecturas, tutorías y el seguimiento de la actitud y participación del alumno en las clases. En la US, debido al gran número prácticas programadas, la calificación final surge de la media de la teoría y las prácticas. Para aprobar la asignatura es necesario superar ambas partes. En la UM el alumno tendrá que superar tanto la parte teórica como la práctica en un $60 \%$ y un $40 \%$, respectivamente. La parte teórica evalúa, además, la asistencia, la participación en clase, la exposición oral y la entrega en tiempo y forma de los comentarios críticos sobre las lecturas propuestas y la entrevista oral final por grupos.

Esta última revisión de los programas refleja una disgregación más notoria de los contenidos y de su localización dentro de la globalidad del plan de estudios de la carrera. Puede notarse claramente, cómo la base de esta asignatura se construye nuevamente siguiendo el modelo utilizado por la carrera de Comunicación Audiovisual. No siempre quedan claramente definidos nuevos objetivos $\mathrm{y}$ ejercicios; tampoco se reformulan o adaptan lo suficiente a un programa diferente y no se observa un temario tecnológico específico. En el caso de la $\mathrm{UAB}$, llama profundamente la atención su posición en el último año de carrera, pero si visualizamos el contenido, comprobamos que es una asignatura de producción y proyectos publicitarios donde el eje tecnológico se concentra en el funcionamiento del sistema de edición digital. Tómese en cuenta que los estudiantes ya han cursado previamente las materias de Lenguaje de Radio y Televisión, y por tanto, poseen la formación suficiente para desarrollar proyectos audiovisuales.

Muy diferente es la propuesta en el resto de facultades donde se ha expor- 
Tabla 7

Inventario de equipamiento tecnológico por universidad

\begin{tabular}{ll}
\hline Universidad & Equipamiento \\
\hline Autónoma de Barcelona & Dalet Plus, Avid, MiniDV \\
Complutense de Madrid & Audio digital, Betacam, DV, Avid \\
País Vasco & Final Cut, AVID \\
Sevilla & Media 100, Final Cut, Betacam, MiniDV, Pro Tools \\
Carlos III & DVD, VHS, Betacam SP, DV, DV-CAM y Mini-DV \\
Málaga & VIVA, Motu Audio 896, Betacam DV \\
Politécnica de Valencia & After Effects, Avid, Mini DV-HD, Adobe Audition \\
\hline
\end{tabular}

Fuente: Información obtenida de las páginas webs institucionales o proporcionada por el personal a cargo de los laboratorios audiovisuales.

Elaboración propia.

tado en su totalidad el modelo nativo de primer y segundo cursos, y se estudian los fundamentos de operación instrumental en diferentes soportes, pero de forma genérica, sin articularlos debidamente con la creación publicitaria. Se enseña, por ejemplo, cómo se monta el trípode, cómo se graba o edita, pero sin explicar de qué manera ese conocimiento sirve para producir un tipo o formato de mensaje publicitario.

Queda claro que para un publicista constituye un saber absolutamente complementario o tangencial el dictado de temas como el entrelazado o transmisión de señales aéreas, digitales o vía satélite (UPV y US), el montaje del cine (US), la producción de un cortometraje de ficción o un programa radial de corte periodístico (UM).

De cualquier forma, la decisión constituye un primer acercamiento a las tecnologías, aun cuando no sea precisando una ligazón técnica directa con su campo habitual de trabajo.
La experiencia directa, en estos casos, demuestra un alto grado de satisfacción del alumno al interactuar con equipamientos, realizando pequeñas piezas, y constituye un complemento muy adecuado para compensar el exceso de sesiones teóricas de los primeros años de la carrera.

\section{Discusión}

La pluralidad de contenidos, metodologías y diseño de prácticas detectada en el análisis de los programas de las titulaciones puede explicarse por la presencia de los siguientes factores:

\section{Unidad responsable}

La orientación de la asignatura y su planeación definitiva está a cargo de un departamento o unidad académica. Son ellos quienes diseñan el programa y la metodología según su adecuación específica al perfil de la carrera, la formación y especialidad del 
profesorado. En función de ello se dispone la carga horaria, su carácter, posición en el plan de estudios y sus baremos de evaluación. Así, por ejemplo, en Comunicación Audiovisual el diseño es más ajustado, la enseñanza en grupos más reducidos y los criterios de evaluación tienen mayor exigencia en comparación con la titulación de Publicidad y Relaciones Públicas, en tanto esta asignatura genera un conocimiento y unas competencias indirectamente vinculadas con su formación troncal. El profesional publicitario no se forma para ser realizador o un experto en la producción de spots; no obstante, debe conocer la esfera creativa, técnica y ha de estar en capacidad de supervisar todas las fases que llevan su elaboración. Igualmente, la formación en ingeniería del profesorado de la Universidad Politécnica de Valencia define la prioridad de las basas físicas o electrónicas para la producción y difusión de señales sobre los temas operativos y en especial de realización de mensajes.

\section{Plan propio o adaptado}

Un segundo factor para definir la congruencia de los temarios es la diferencia entre programa propio o adaptado. En el primer caso se percibe una perfecta concordancia metodológica entre la teoría, la práctica y los procedimientos de evaluación. Las unidades didácticas siguen a menudo la lógica estructural de los libros de referencia de la especialidad (Kindem y otros 2007; Zettl 2006; Lugmayr y otros 2004; Martínez 2004, 2000; Sainz 1995). En programas adaptados o reproducidos de Comunicación Audiovisual, el planteamiento termina siendo más general y en algunos casos superficial. No se abordan debidamente contenidos fundamentales, los ejercicios carecen de especificidad y relación, y faltan revisión y actualización bibliográfica. Los ajustes a estas deficiencias se aprecian en la titulación de Publicidad y Relaciones Públicas, adaptada recientemente al Plan Boloña. La necesidad obligada de reformular el modelo de nota de teoría al $50 \%$ ha impulsado una muy considerable producción de recursos audiovisuales e interactivos a medida, para preparar y corregir las prácticas de campo. Esta nueva didáctica, además, intenta subsanar la disminución de la teoría directa con materiales impresos y tutorías periódicas individuales y grupales presenciales o mediante las plataformas virtuales de enseñanza interactiva vía internet.

\section{Carga horaria}

Otra variable de influencia sustancial para un desarrollo eficaz del temario teórico y práctico es la carga horaria. Mientras más horas por semana y en el semestre o año se dedica a la asignatura, mayor número de contenidos pueden desarrollarse en profundidad y se logra implementar un modelo preciso y personalizado para las prácticas . Sin embargo, el efecto global de 
dichas diferencias no siempre genera resultados positivos. Un exceso en el número de horas por semana y la duración total puede acabar saturando tanto al alumno como al profesor, perjudicando en definitiva la calidad de la docencia y el alcance de los objetivos de aprendizaje. A los profesores probablemente les resulte cansino explicar un mismo protocolo y método de evaluación varias veces durante el curso. Se ha constatado que el exceso de horas a lo largo del período lectivo lleva fácilmente a incluir, por ejemplo, prácticas de rodaje o producción, sin haberse impartido los conocimientos suficientes de lenguaje, géneros y narrativas, los cuales corresponden a asignaturas posteriores de cualquiera de las titulaciones. Esto puede obedecer a una decisión de incentivar la motivación creativa del estudiante, empleando una importante cantidad de horas de la programación dedicadas al trabajo práctico.

\section{Complementariedad teórico-práctica}

Pero la adecuación y el ensamble de los equipos docentes no solo se valoran por la generación de un buen programa teórico y su conexión implícita para la formación de competencias conceptuales concretas, sean estas en el plan de estudios tradicional o en el actual. En tanto asignatura eminentemente práctica, es vital para la enseñanza de las tecnologías la estructura logística existente: volumen, características técnicas y funcionales de los equipos de laboratorio. Por tanto, el sistema pedagógico en este caso genera un proceso retroactivo dependiente, donde la necesaria concordancia teórico-práctica y el diseño de los ejercicios de taller correspondientes se planifican recurriendo al soporte de áreas tecnológicas diferentes. La vocación interdisciplinaria de la asignatura se hace evidente cuando se incluyen, por ejemplo: temarios cinematográficos de alta performance, estudio y análisis de procesos digitales complejos, de iluminación o videoarte, los cuales, en última instancia, solo son desarrollados teóricamente por falta de equipo especializado.

\section{Grupos y equipamiento}

Un último factor de las diferencias en la pluralidad de estructuras y módulos es que todos los modelos de asignatura finalmente se organizan según la disponibilidad, número de espacios y la cantidad de equipos del centro. Los grupos deben formarse y distribuirse para instalarse en módulos, estaciones o unidades de trabajo, con supervisión de profesores tutores. Por norma general, los alumnos disponen de un tiempo para realizar las prácticas, limitado a las horas de clase, porque fuera de este horario los equipos están reservados por profesores o alumnos de otras materias y titulaciones. Este planteamiento de fragmentación/rotación genera tiempos de inmersión tecnológica completamente desiguales para los alumnos. Consi- 
derando el número elevado de estudiantes por curso en las universidades públicas de España, la planificación de grupos para las prácticas es extremadamente cuidadosa, funcional y tiene la ventaja de poderse reformular año a año, para convertirse en un modelo de trabajo eficiente y personalizado dentro y fuera de su contexto inmediato. En las universidades privadas este problema no suele presentarse tan a menudo, pues, al haber por lo general más espacios y menos estudiantes, es posible formar pocos grupos con menos alumnos cada uno y asignar un mayor número de horas para la formación práctica. La desventaja de la actualización o renovación tecnológica debe asumirse como una variable y tiene que ser controlada debidamente por quienes en la práctica llevan a cabo los planes de estudio, aplicando políticas de organización y planificación eficientes junto con el profesorado, para mejorar e innovar la calidad de su docencia. De no ser así el nivel de la educación puede verse afectado en perjuicio directo de los alumnos.

\section{Conclusiones y reflexión final}

El análisis curricular comparativo ha proveído un diagnóstico del estado actual de la cuestión de la asignatura de Tecnologías. Se ha constatado la existencia de planteamientos que responden a diversas perspectivas y prioridades respecto de los contenidos por impartir. Existen razones para entender la opción de los centros: posicionamiento institucional y de mercado, formación y especialización de la plantilla docente, volumen, característica y distribución de la infraestructura tecnológica de los centros, entre otros.

El exceso de expectativas de los alumnos por formación práctica en el entorno audiovisual y los requerimientos del mercado, parecen influir en su carácter, los contenidos y el diseño de los ejercicios. Es necesario adoptar definitivamente un posicionamiento al respecto, porque se está cayendo en el riesgo de formar capacidades y destrezas operativas más propias de un profesional técnico, de mando medio y no universitario. Pero esta evidente pluralidad de ofertas en torno a la asignatura, extensiva a las carreras de Comunicaciones en general, no debe interpretarse per se, como un indicador de incongruencia interna, sino mejor, como el abanico de alternativas formativas disponible, donde el estudiante puede bien analizar y comparar, ajustándolas a sus expectativas e intereses específicos de aprendizaje, antes de tomar la decisión de qué carrera desea realizar.

La investigación reconoce mayor coherencia, pertinencia, sentido secuencial y exigencia respecto del dominio instrumental en la titulación de Comunicación Audiovisual, en comparación con las de Periodismo y $\mathrm{Pu}$ blicidad, y Relaciones Públicas. No obstante, en esta última, las experiencias de la Prueba Piloto son positivas 
y aplicables a la mejora de las demás asignaturas.

Se sugieren para ello las siguientes recomendaciones:

- Elaboración de programas ad-hoc: El avance reciente y los modelos tecnológicos existentes en la dinámica profesional de las tres titulaciones, debe reflejarse claramente en los nuevos planes de estudio. Las ofertas deben centrarse en contenidos específicos para cada carrera, en planteamientos estructurales nativos y rutinas de trabajo para cada medio de comunicación. La metodología debe diseñarse en función de estos elementos para transformarlos en un diseño e instrumentos de seguimiento y evaluación eficientes y a medida. Se debe poner fin a la etapa de planes adaptados, precisamente por una presencia excesiva de temarios innecesarios y disonancias teórico-prácticas, finalmente inadecuados para la formación profesional.

- Rediseño del sistema de prácticas: Interconectando directamente las rutinas de producción con los ejercicios tecnológico-operativos. Los beneficios de esta implementación logística se traducen en dinámicas individuales y grupales más eficientes, ejercicios puntuales y ajuste preciso de competencias, aplicables a la totalidad de asignaturas prácticas de las tres carreras.

- Mayor subdivisión de tareas, grupos y espacios: El esquema de fragmenta- ción por grupos es indispensable para el conocimiento de la tecnología, pero obliga a poner en marcha propuestas concretas para hacer explicaciones especializadas en cada espacio y resolver los entrampamientos puntuales de grupos ubicados en una o varias zonas de trabajo. Es indispensable un aumento de la plantilla docente e incluso la incorporación de asistentes especializados. Los beneficios de este modelo se traducen en: mayor tiempo de permanencia del alumno y de grupos en cada espacio de prácticas, rotación evolutiva de grupos, incremento potencial de inmersión tecnológica, mejora de los mecanismos de enseñanza personalizada y supervisada, seguimiento preciso del aprendizaje y obtención de indicadores más fiables de la evolución del alumno para la evaluación.

- Trabajo multidisciplinario: El soporte humano especializado es indispensable y forma parte del núcleo de funcionamiento de la asignatura. Es esencial el auxilio de los técnicos, los ingenieros y los especialistas informáticos para resolver problemas operacionales de conexión, compatibilidad de formatos, códecs o para el control de opciones avanzadas de los dispositivos. Sin embargo, el esfuerzo por la actualización docente constituye un mecanismo muy valioso para ir desligando esta dependencia progresivamente. 
- Participación y motivación docente: Resulta impostergable informar acerca de los cambios estructurales y las ventajas del nuevo modelo europeo. El sistema de trabajo parte ahora de una labor coordinada entre académicos y el mundo profesional, donde el énfasis práctico impone reformular la cuota teórica y reflexiva sin perder sus niveles mínimos. Esta coyuntura deja asomar algunas alternativas interesantes, que van desde la modificación de los niveles de practicidad de las carreras, el aumento de los presupuestos globales para compra de equipamiento y contratación docente, la capacitación y actualización docente para adentrarlo en las nuevas tecnologías, entre otras. Estas medidas permitirán, de cara al futuro, mejorar las propuestas pedagógicas y la eficacia de los diferentes mecanismos docentes para desarrollar un método de trabajo orientado a la personalización, cuyos efectos irán en beneficio directo de la calidad de la enseñanza y el aprendizaje de los estudiantes de este tipo de asignaturas.

\section{Bibliografía}

Aguaded Gómez, José Ignacio (1996). Comunicación audiovisual en una enseñanza renovada. Propuestas desde los medios. Huelva: Grupo Pedagógico Andaluz “Prensa y Educación.
Aneca. Libro blanco de los títulos de grado de comunicación [en línea].<http://www.aneca.es/media/ 150336/libroblanco_comunicacion_def.pdf $>$.

Cabero, J.; Castaño, C.; Cebreiro, B.; Gisbert, M.; Martínez, F.; MoraLes, J. A.; Prendes, M. P.; Romero, R. y J. SAlinas (2003). “Las nuevas tecnologías en la actividad universitaria". Pixel-Bit. Revista de Medios y Educación 20 [en línea]. <http:// tecnologiaedu.us.es/bibliovir/pdf/ nnttact.pdf $>$.

Cabrera K. y L. González (2006). Currículo universitario basado en competencias. Barranquilla: Ediciones Uninorte.

FANDOS GARRIDO, Manuel (2009). Las tecnologías de la información y la comunicación en la educación: Un proceso de cambio. Barcelona: Recerca II, Universitat Rovira i Virgili.

Kindem Gorham, Anders (2007). Manual de producción audiovisual digital. Barcelona: Omega.

Lugmayr, A.; NiIRAnen, S. y S. Kalli (2004). Digital Interactive TV and Metadata. Nueva York: Future Broadcast Multimedia, Springer.

Martínez Abadía, J. (2004). Manual básico de tecnología audiovisual y técnicas de creación, emisión y difusión de contenidos. Barcelona: Paidós.

- (2000). Introducción a la tecnología audiovisual: televisión, vídeo, radio. Barcelona: Paidós. 
Ministerio de Educación de España. Pautas generales sobre el nuevo Modelo Europeo de Educación [en línea]. $<$ http://www.queesbolonia.es/>.

RodríGuez EsCANCIANO, Imelda (ed.) (2009). El nuevo perfil del profesor universitario en el EEES, claves para la renovación metodológica. Valladolid: Universidad Europea Miguel de Cervantes.

SAINZ, Miguel (1995). Manual básico de producción en televisión. Madrid: IORTV.

Varias universidades. Planes de estudio y programas oficiales en vigor de la asignatura de Tecnologías [en línea].

Universidad Autónoma de Barcelona $<$ http://ccc-web.uab.es/index. php $>$.

Universidad Carlos III. <http://www3. uc3m.es/reina/Fichas/fichas_1/ 21113511.html>.

Universidad Complutense de Madrid $<$ https://ucmnet.ucm.es/oferta/es/a nyo200910/asignaturas/plan001X/a sig105593.html>.

_- <https://ucmnet.ucm.es/oferta/ es/anyo200910/asignaturas/plan00 1T/asig105235.html>.
Universidad de Málaga. <http://hs. sci.uma.es:8070/ht/2009/ProgramasAsignaturas_Titulacion_102_AsigUMA_7610. pdf $>$.

_. $\quad<$ http://hs.sci.uma.es:8070/ht/ 2009/ProgramasAsignaturas_Titulacion_104_AsigUMA_7496.pdf>.

—. $\quad<$ http://hs.sci.uma.es:8070/ht/ 2009/ProgramasAsignaturas_Titulacion_103_AsigUMA_7436. pdf >.

Universidad de Santiago de Compostela. <http://www.usc.es/gl/centros/cc_comunicacion/materia.jsp? materia $=36855 \& a n o=60>$.

—_. <http://www.usc.es/gl/centros/cc_comunicacion/materia.jsp? materia $=36773 \& a n o=60>$.

Universidad de Sevilla. <http://www. fcom.us.es/images/stories/ordenacion/Programas/titulaciones/CAV/ 2008/1/tecnologia_de_los_medios_ audiovisuales.pdf $>$

Universidad Politécnica de Valencia. $<$ http://www.upv.es/pls/oalu/ sic_asi.Busca_Asi?p_codi $=5747 \& p$ _caca $=$ act\&P_IDIOMA $=c \&$ p vista $=>$.

Zettl, H. (2006). Television Production Handbook, 9. a edición. San Francisco: San Francisco State University Casebound. 